\title{
TENSION FREE ONLAY VENTRAL HERNIA REPAIR BY POLYPROPYLENE MESH- A NEWER APPROACH
}

\author{
${ }^{1}$ Associate Professor, Department of Surgery, SCBMCH, Odisha. \\ ${ }^{2}$ Assistant Professor, Department of Surgery, VIMSAR, Burla, Odisha. \\ ${ }^{3}$ Postgraduate Student, Department of Surgery, VIMSAR, Burla, Odisha. \\ ${ }^{4}$ Postgraduate Student, Department of Surgery, VIMSAR, Burla, Odisha. \\ ${ }^{5}$ Postgraduate Student, Department of Surgery, VIMSAR, Burla, Odisha.
}

Jayanta Kumar Biswal', Malaya Krishna Nayak², Dharbind Kumar Jha33, Bikash Chandra Pal', Itishree Das ${ }^{5}$

ABSTRACT

\section{BACKGROUND}

Ventral Hernia repair remains one of the most commonly performed surgical procedure. Operations for ventral hernia were notorious, because of high failure rate. With continued improvement of the materials and operative procedure, there is decrease in morbidity and overall recurrence rate.

\section{MATERIALS AND METHODS}

66 patients of ventral hernia of different size were included in the study during the study period from November 2011 - October 2016. The patients were examined clinically and the diagnosis of ventral hernia was confirmed by ultrasonography. Then after proper preoperative evaluation and preparation, the onlay polypropylene mesh repair was done over mobilised anterior rectus sheath sutured in midline. Then, the patients were followed postoperatively for complications up to 5 years.

\section{RESULTS}

In most of the cases, there was no postoperative complication and no mortality. Only 5 patients have minimal wound infections, which were managed by conservative treatment successfully. One patient had residual pain till the 2nd week, which was subsided by oral analgesics. During this 5 years of study period, followup with 6 months interval there was no report of residual pain or recurrence.

\section{CONCLUSION}

We had utilised a method of tension-free onlay polypropylene mesh repair over the mobilised anterior rectus sheath sutured in the midline over the hernia defect. It is very easy to perform even by a general surgeon. The procedure is safe without any morbidity, mortality and recurrence.

\section{KEYWORDS}

Ventral Hernia, Polypropylene Mesh, Tension-Free Onlay Repair, Anterior Rectus Sheath.

HOW TO CITE THIS ARTICLE: Biswal JK, Nayak MK, Jha DK, et al. Tension free onlay ventral hernia repair by polypropylene mesha newer approach. J. Evolution Med. Dent. Sci. 2017;6(46):3590-3594, DOI: 10.14260/Jemds/2017/774

\section{BACKGROUND}

By definition, Hernias of anterior abdominal wall or ventral hernias represent defects in the parietal abdominal wall fascia and muscle through which intra-abdominal or peritoneal contents can protrude.1,2

Ventral hernia repair is a common procedure in the general surgery, which is notorious because of high failure rate. Reoperation rates are as high as $12.3 \%$ at 5 years and $23 \%$ in 13 years. 3,4 Ventral hernias are an established complication following laparotomy. ${ }^{1-9}$ Incisional hernias pose difficult reconstructive challenges, and are often fraught with high recurrence rates and postoperative complications. Up to 67 percent of ventral herniorrhaphy repairs recur and complications including seroma or haematoma formation, surgical site infection and dehiscence are reported in up to 75 percent of cases.1-9

Financial or Other, Competing Interest: None.

Submission 01-05-2017, Peer Review 28-05-2017,

Acceptance 02-06-2017, Published 08-06-2017.

Corresponding Author:

Dr. Malaya Krishna Nayak,

No. 3R/21, Doctors Colony,

Burla-768017, Odisha.

E-mail: drmalayanayak@gmail.com

DOI: $10.14260 /$ jemds $/ 2017 / 774$
This is in contrast to other reports that have demonstrated much lower rates of deleterious events postoperatively. This variability suggests that patient selection and operative technique can be optimised to reduce complications and improve outcomes. Understanding differences in surgical technique is essential for achieving these goals. It has been demonstrated that 70 to 85 percent of hernia repairs use some type of surgical mesh to reduce recurrence rates following fascia repair. Despite its ubiquitous use, there is no ideal technique described to standardise mesh placement during abdominal wall reconstruction. The specific location of mesh placement used to reinforce the repair is one variable that remains largely contested. The most common methods of mesh placement include onlay, interposition, retrorectus and underlay graft. The ideal location for mesh placement in ventral hernia repair remains unclear. ${ }^{9}$

So the type of repair should be well chosen as well as the materials and should be meticulously performed. The factors to be considered are adequate preoperative preparations, anatomical dissection, repair without tension and use of well accepted, non-absorbable, non-irritant implant and effective postoperative management.5,6,7 Keeping in view of the above facts, a new approach of tension-free onlay ventral hernia repair by polypropylene mesh was conducted.6,7,8 


\section{MATERIALS AND METHODS}

\section{Study Design}

Prospective Study

Tension free onlay ventral hernia repair by polypropylene mesh is a standard procedure done by us in our institution. This study was conducted in the Department of General Surgery, VIMSAR, Burla and Odisha during the period from November 2011 - October 2016. Total 66 patients of ventral hernia were included in the study.

\section{Exclusion Criteria}

1. Incisional hernias that had not crossed one year after the operation causing it.

2. Small ventral hernia, less than $2 \mathrm{~cm}$ in diameter.

3. Patient not willing for mesh repair.

After proper history taking, physical examination, routine investigations and correction of predisposing factors, the patients were prepared for surgical interventions.

\section{Operative Procedure}

The surgery was done with full abdominal wall relaxation by general or spinal anaesthesia. Old scar and subcutaneous tissue were dissected away from the hernial sac. Isolation of healthy tissue was done all around, $6-7 \mathrm{~cm}$ from the margin of the defect (Figure 1). Abdominal cavity was entered through a virgin area or directly through the hernia and all adhesions were taken down from the facial defect. Two longitudinal incisions were made over each of the anterior rectus sheath, $3-4 \mathrm{~cm}$ away from its medial edges, exposing the underlying rectus muscle (Figure 2).

The incisions were extended up and down over the entire length of the hernia defect.

Both the medial flaps of the anterior rectus sheath were mobilised from the rectus muscle and brought to the midline for apposition and closure of hernial defect. Closure was done with $(1-0)$ polypropylene by continuous suture, forming virtually a new linea alba without any facial tension (Figure 3).

A propylene mesh of required size $(5-6 \mathrm{~cm})$ larger than the margin of entire defect was put over the closed hernia defect, exposed rectus muscle and intact lateral flaps of the anterior rectus sheath. The margins of the mesh were fixed by interrupted (1 - 0) polypropylene stitches along with a few stitches in the midline to the newly formed linea alba (Fig. 4). The skin and the subcutaneous fat was closed by interrupted polyamide mattress sutures over two suction drains. The drains were maintained at continuous suction till some amount of serous discharge was there (usually 4 - 5 days).

Skin stitches were removed around 8 to 10 days. Patients were advised to use abdominal support and avoid strenuous abdominal exercise for about 3 months. All the patients were kept under followup with 2 months interval up to 6 months and then at 6 months interval for the results and complications.

\section{RESULTS}

In our study, hernias taken were larger than $10 \mathrm{~cm}$ in diameter in 6 patients $(9.09 \%)$, medium size $(6-10 \mathrm{~cm})$ in 21 patients $(31.8 \%)$ and small size $(2-6 \mathrm{~cm})$ in 39 patients (59.09\%).

\begin{tabular}{|c|c|c|}
\hline $\begin{array}{c}\text { No. of Days of } \\
\text { Analgesic Required }\end{array}$ & No. of Patients & Percentage \\
\hline One & 66 & $100 \%$ \\
\hline Two & 60 & $91 \%$ \\
\hline Three & 32 & $48 \%$ \\
\hline Four & 6 & $9 \%$ \\
\hline Five & Nil & Nil \\
\hline Table 1. Postoperative Pain and Analgesics \\
\hline
\end{tabular}

Tramadol $100 \mathrm{mg}$ or diclofenac $50 \mathrm{mg}$ IM was administered to all the patients on day 1 , which was not necessary at the end of day 5 . All the patients were able to perform their personal routine works by day 5 (Table 1).

\begin{tabular}{|c|c|c|}
\hline Post-Operative Period & No. of Cases & Percentage \\
\hline 2 weeks & 48 & $73 \%$ \\
\hline 3 weeks & 14 & $21 \%$ \\
\hline 6 - 8 weeks & 4 & $6 \%$ \\
\hline \multicolumn{2}{|c|}{ Table 2. Return to Active Life } \\
\hline
\end{tabular}

$48(73 \%)$ patients mostly belonging to executive, office bearer and sedentary workers were able to resume full activity by the second week, next 14 patients by the end of $3^{\text {rd }}$ week. Rest of 4 patients who were mostly labourers took 6 - 8 weeks to resume their full activity (Table 2).

\begin{tabular}{|c|c|c|c|c|c|}
\hline Duration & $\begin{array}{c}\text { No. of } \\
\text { Cases }\end{array}$ & DVT & $\begin{array}{c}\text { Haematoma/ } \\
\text { Seroma }\end{array}$ & $\begin{array}{c}\text { Wound } \\
\text { Infection }\end{array}$ & $\begin{array}{c}\text { Residual } \\
\text { Pain }\end{array}$ \\
\hline $1^{\text {st }}$ week & 66 & 0 & 0 & 5 & 3 \\
\hline $2^{\text {nd }}$ week & 66 & 0 & 0 & 0 & 1 \\
\hline \multicolumn{7}{|c|}{ Table 3. Post-Operative Complications } \\
\hline
\end{tabular}

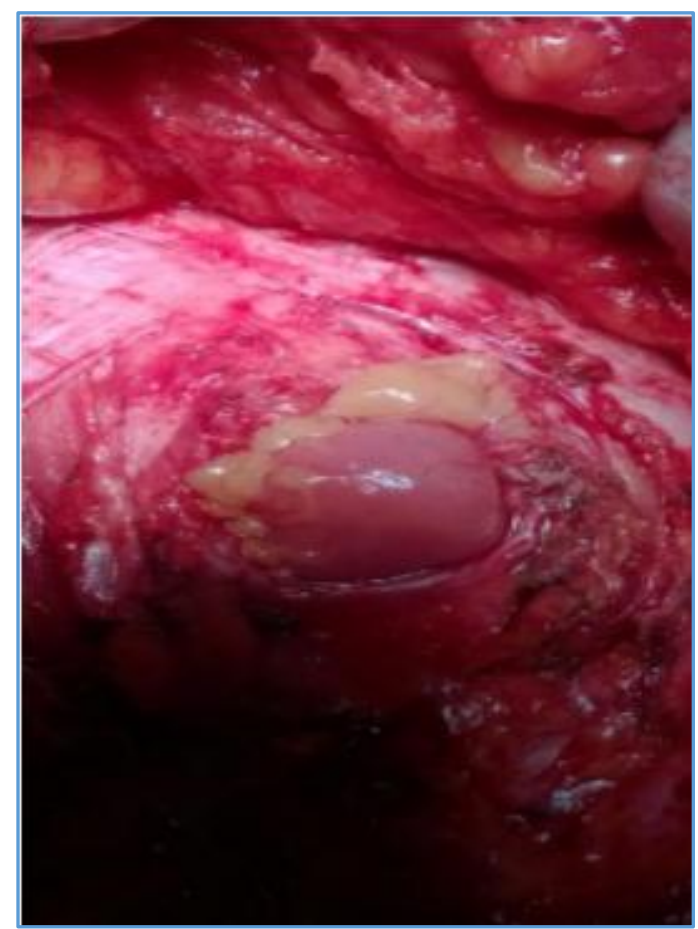

Figure 1. Showing the Dissected Ventral Hernia 


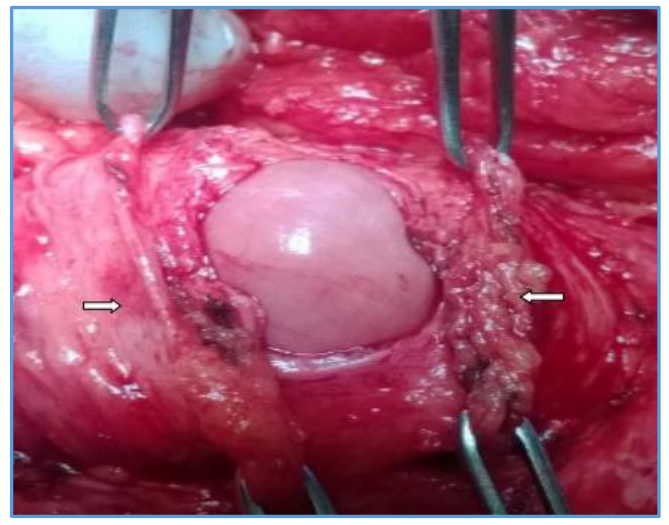

Figure 2. Longitudinal Incision over both Anterior Rectus Sheath

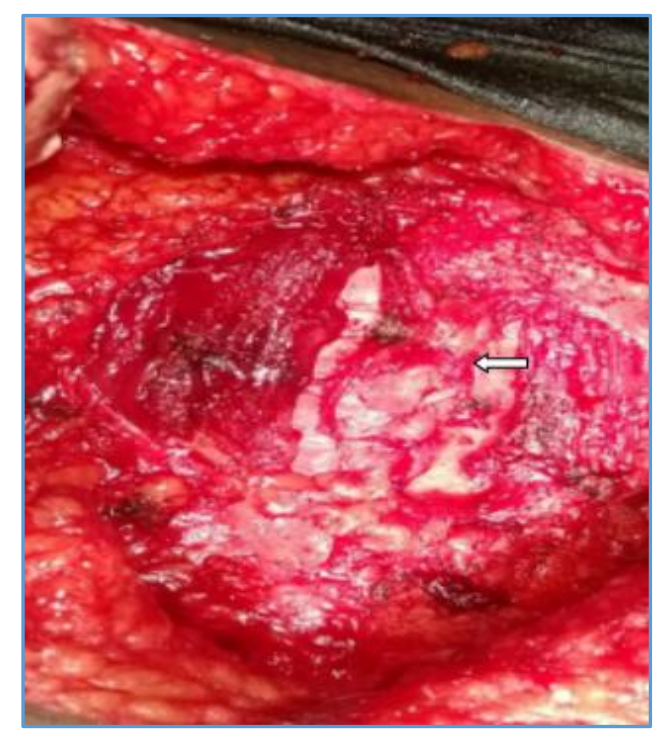

Figure 3. Showing both Rectus Sheath Mobilised to the Midline and Sutured. Exposed Rectus Muscle on Both Sides

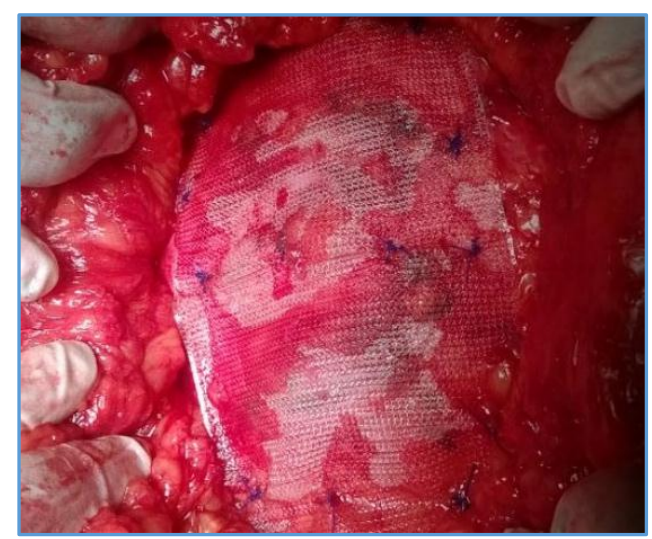

Figure 4. Showing Onlay Prolene Mesh after Fixation

In most of the cases, there was no post-operative complication and there was no mortality. Only 5 patients have minimal wound infections, which were managed by conservative treatment successfully. One patient had residual pain till the $2^{\text {nd }}$ week, which was subsided by oral analgesics (Table 3).

During these 5 years of study period, followup with 6 months interval, there was no report of residual pain or recurrence.

\section{DISCUSSION}

In this descriptive study, 66 cases of ventral hernia, of the defect size more than $2 \mathrm{~cm}$ were studied by definition. "Hernias of anterior abdominal wall or ventral hernias represent defects in the parietal abdominal wall fascia and muscle through which intra-abdominal or peritoneal contents can protrude."

Primary open ventral hernia repair is based on suture approximation of Aponeurosis on each side of the hernia defect. However, recurrence rates after this procedure range from $41 \%$ to $52 \%$ in the long term. The introduction of prosthetic meshes in hernia repair has reduced recurrence rates. Prosthetics used for Ventral Hernia Repair are broadly classified into 3 categories- Synthetic polymers, composites and biologic prosthetics. Polypropylene is the most commonly used mesh for hernia repair with more than 1 million prosthetics implanted each year. It is a hydrophobic polymer produced by the polymerisation of propylene, a derivative of propane gas. Its rough surface prevents any migration within host tissue. It is extremely resistant to biologic degradation, as it is not significantly weakened by tissue enzymes. It can be either woven or knitted and is formulated as a monofilament or in a dual or multifilament arrangement. Polypropylene is typically knitted, because this arrangement allows for increased porosity, flexibility and identical mechanical properties in all directions. In recent years, however, lightweight polypropylene meshes have emerged that differ significantly from heavyweight mesh in regard to pore size, thickness, tensile strength and host response. ${ }^{10-11}$ Klinge et al also compared the elasticity of the abdominal wall in human cadavers to that of the heavyweight and lightweight polypropylene meshes. Universally, the human abdominal wall had significantly greater elasticity than polypropylene, although the lightweight mesh mimicked human tissues more closely than the heavyweight polypropylene. In addition, patients in whom hernias were repaired with heavyweight, Prolene reported chronic pain and a "stiff abdomen" more frequently than those patients whose hernias were repaired with Vypro, a lightweight polypropylene mesh in the past several years, biologic prosthetics have been introduced for ventral hernia repair. These prostheses are advantageous, in that they may be used intraperitoneally or extraperitoneally; however, they are generally expensive and have not proven a cost-effective solution for uncomplicated ventral hernia repair. Synthetic mesh remains a cost-effective, durable solution for most Ventral Hernia Repair. ${ }^{10-11}$ Lightweight polypropylene mesh has recently proven a comfortable and affordable option for mesh for ventral hernia repair. The ideal synthetic mesh possesses 8 characteristics: (1) No carcinogenic, (2) Chemically inert, (3) Resistant to mechanical strains, (4) Capable of being sterilised, (5) Inert to body and tissue fluids, (6) Capable of limiting foreign body reaction in the host, (7) Amenable to fabrication in the necessary form, and (8) Unlikely to produce allergy or hypersensitivity reactions. With the introduction of bioprosthetic mesh, additional criteria for the ideal prosthetic have been proposed. Namely, the material must resist infection, provide a barrier to adhesions on the visceral side and must respond in vivo similarly to autologous tissue. ${ }^{10-11}$ 
However, the mesh repairs require wide dissection of soft tissue, which contributes to an increased incidence of woundrelated complications. ${ }^{1}$ Among the open repairs, the onlay technique was the most widely used one. In this method, a polypropylene mesh is sutured onto the anterior rectus sheath. The procedure is easy to perform, but it has a considerable morbidity rate and recurrence rate and therefore is not used often at present. ${ }^{1}$

In the inlay technique, the mesh is sutured to the margin of the Aponeurosis. This technique has been used to cover large Aponeurotic gaps and it carries extremely high recurrence rates. ${ }^{1}$ In the extraperitoneal underlay technique, the mesh is placed retromuscularly and preperitoneally as described by Stoppa in 1989. This technique requires limited soft tissue dissection; therefore, it carries low morbidity and recurrence rates. ${ }^{1}$

The intraperitoneal underlay technique was introduced by McCarthy and Twiest in 1981. First, they used polypropylene mesh, which was sutured to the peritoneal edge of the hernia sac. However, polypropylene caused the formation of adhesions to the adjacent bowel loops and was replaced by the expanded polytetrafluoroethylene (ePTFE) mesh or bilayer PTFE and polypropylene mesh. Millikan et al applied this technique by using full-thickness transfascial sutures in 102 patients with a $0 \%$ recurrence rate in a median followup of 28 months. This approach has been regarded as the gold standard for ventral hernia repair by the American Hernia Society. By placing the mesh posterior to the abdominal wall, the underlay technique applies Pascal's law, which states that any pressure exerted on an enclosed fluid is transmitted undiminished throughout the fluid and acts equally in all directions. Therefore, as the intraabdominal pressure increases, equal amounts of force are exerted across the mesh. On the contrary in the onlay technique, any increase in intra-abdominal pressure exerts lifting forces against the mesh. Consequently, the underlay repair seems mechanically advantageous. ${ }^{1}$

The relatively high morbidity and recurrence rates of the open repair techniques prompted the development of the laparoscopic approach in an effort to improve the clinical outcome. The laparoscopic repair follows the principles of the open underlay repair and has the same advantages.

A laparoscopic ventral hernia repair of a large hernia defect $(>8 \mathrm{~cm})$ does not achieve satisfactory muscular strength, and an open repair with open/laparoscopic component separation technique may be a more functional repair for the patient. Component separation technique is a natural method of fascia-fascia closure without the complication of an artificial implant caused by creation of a linea alba, which provides a midline anchor. ${ }^{1}$ This repair allows for advancement of the rectus abdominis muscle up to $10 \mathrm{~cm}$ per side, facilitating closure of large gaps of the abdominal wall. A recently introduced endoscopic technique with the use of a balloon dissector in the space between the external oblique and internal oblique muscles and laparoscopic division of the external oblique Aponeurosis along the midclavicular line may reduce substantially the abdominal wall wound morbidity associated with the component separation technique. However, this technique achieves only $86 \%$ of the abdominal wall advancement that is obtained with the open technique. ${ }^{1}$
The posterior component separation technique has also been introduced as a modification of the Rives-Stoppa technique allowing for significant posterior rectus fascia advancement, wide lateral dissection and a large space for mesh sublay. Transversus abdominis muscle release is a novel approach to posterior component separation technique for the repair of complex abdominal hernias as well as the repair of recurrent hernias after use of the anterior component separation technique. According to this technique, the posterior rectus sheath and the underlying transversus abdominis muscle are incised and the lateral space is developed. The dissection proceeds to the accurate line of Douglas toward the space of Retzius. The posterior rectus sheaths are approximated in the midline, and the mesh is placed in the retromuscular space and fixed to the abdominal wall with sutures. This technique is an important addition to the armamentarium of surgeon's undertaking abdominal wall reconstructions and represents the gold standard of open repair according to the American Hernia Society. ${ }^{1}$

Though open or laparoscopic underlay mesh repair is the gold standard for ventral hernia, it is costlier and hence not affordable by poor patients. So here we tried an alternative method of anatomical fascial repair with tension free mesh placement that serves the purpose and is also cost effective and very easy to do with onlay polypropylene mesh after mobilising anterior rectus sheath and suturing them in the midline.

66 cases of ventral hernia of the defect size more than 2 $\mathrm{cm}$ were taken up for the studies. All cases were repaired with onlay polypropylene mesh after mobilising anterior rectus sheath and suturing them in the midline. With the usual postoperative management, only 5 patients have wound infection and one had residual pain over 2 weeks which could be managed conservatively. Most of the patients could resume their active physical work at the end of second week $(73 \%)$ and rest within 8 weeks. During this period of followup in 6 months interval, there was no report of residual pain or recurrence. All these benefits are the effect of proper anatomical fascial repair followed by tension free proper mesh placement.

The patient suffered from less post-operative pain, because of the tension free repair. There was no recurrence rate during this study period, because of proper tension free anatomical repair and the use of polypropylene mesh providing additional strength.

\section{CONCLUSION}

Repair of ventral hernias with mesh as opposed to suture has substantially improved long-term outcomes and is accepted as the standard of care. However, many studies demonstrate an increased risk for wound complications with mesh placement including infections, seromas and mesh erosions. The risks of these complications are affected by where the mesh is placed. For example, mesh exposed to intraabdominal contents potentially increases the risks of adhesions, bowel obstruction and fistula formation. While repair of ventral hernias with mesh is considered routine, there is no consensus on the best location to place the mesh. ${ }^{10-12}$

When considering the best location for mesh placement, a number of features are important to consider. First, mesh 
tissue integration may reduce long-term recurrence with theoretically improved rates with greater mesh-tissue overlap. Second, wound complications increase the risk of recurrence. Thus, techniques that avoid the development of DE vascularising flaps may be preferred. Third, the ideal mesh placement should have tissue coverage to minimise exposure to superficial SSIs as well as intra-peritoneal contents. Last, technical ease may affect surgeon's choice of procedure as well as risks for postoperative complications. Sublay repair allows for tissue integration from two loadbearing tissues from both sides- Posterior rectus sheath and the anterior myofascial complex.10-12 In addition, sublay mesh placement protects the mesh from exposure from superficial wound complications, intra-abdominal adhesions and contamination. Creation of DE vascularising skin flaps is avoided. Alternatively, inlay repair does not allow for any tissue integration, any wound complication can potentially expose the mesh and the mesh is exposed to intra-abdominal contents. While onlay allows for tissue ingrowth from two directions, the skin flaps are not loadbearing. Mesh placed in the onlay location is vulnerable forcing the surgeon to create DE vascularising skin flaps and leaving the mesh susceptible to superficial wound complications. Underlay has the advantages of protecting the mesh from superficial wound complications and avoiding development of skin flaps. However, this position requires mesh to be placed intraabdominally, which leaves the mesh. In addition, risks of damaging the blood supply, muscle or lateral penetrating nerves pose technical concerns. ${ }^{10-12}$

So in our study, we had utilised a method of tension-free onlay lightweight polypropylene mesh repair over the mobilised anterior rectus sheath sutured in the midline over the hernia defect. Here, we are restoring anatomical fascial plane and also reconstructing a new linea alba and reinforcing the defect with a lightweight polypropylene mesh without tension. Here, we are not damaging the neurovascular supply and also not coming with contact of intraperitoneal contents and hence the chances of adhesion, devascularisation is avoided.

It is very easy to perform even by a general surgeon. The procedure is safe without any morbidity, mortality and recurrence. We recommend this procedure as of choice for ventral hernia repair. The time period of our study is only 5 years. So, more time period of study should be considered before taking up of this procedure as a procedure of choice.

\section{REFERENCES}

[1] Misiakos EP, Patapis P, Zavras N, et al. Current trends in laparoscopic ventral hernia repair. JSLS 2015;19(3):pii: e2015.00048.

[2] Nieuwenhuizen J, Kleinrensink GJ, Hop WC, et al. Indications of incisional hernia repair: an international questionnaire among hernia surgeons. Hernia 2008;12(3):223-5.

[3] Flum DR, Horvath K, Koepsell T. Have outcomes of incisional hernia repair improved with time? A population-based analysis. Ann Surg 2003;237(1): 129-35.

[4] George CD, Ellis $\mathrm{H}$, et al. The results of incisional hernia repair: a twelve year review. Ann R Coll Surg Eng 1986;68(4):185-7.

[5] Lemount PM, Ellis H. Incisional hernia in re-opened abdominal incisions: an overlooked risk factor. $\mathrm{Br}$ J Surg 1988;75(4):374-6.

[6] McCarthy JD, Twiest MW. Intraperitoneal polypropylene mesh support of incisional hermiorrhaphy. Am J Surg 1981;142(6):707-11.

[7] Molloy RG, Moran KT, Waldron RP, et al. Massive incisonal hernia: abdominal wall replacement with Martex mesh. Br J Surg 1991;78(2):242-4.

[8] Schumpelick V, Klinge U, Junge $K$, et al. Incisional abdominal hernia: the open mesh repair. Langenbecks Arch Surg 2004;389(1):1-5.

[9] Albino FP, Patel KM, Nahabedian MY, et al. Does mesh location matter in abdominal wall reconstruction? A systematic review of the literature and a summary of recommendations. Plast Reconstr Surg 2013;132(5):1295-304.

[10] Weber G, Baracs J, Horvath OP. "Onlay" mesh provides significantly better results than "sublay" reconstructtion. Prospective randomized multicenter study of abdominal wall reconstruction with sutures only, or with surgical mesh-results of a five-years follow-up. Magy Seb 2010;63(5):302-11.

[11] Shankaran V, Weber DJ, Reed RL, et al. A review of available prosthetics for ventral hernia repair. Ann Surg 2011;253(1):16-26.

[12] Holihan JL, Nguyen DH, Nguyen MT, et al. Liang mesh location in open ventral hernia repair: a systematic review and network meta-analysis. World J Surg 2015. 